\title{
Retinopatia diabética em pacientes de um programa de atendimento multidisciplinar do Hospital das Clínicas de Ribeirão Preto - USP
}

\author{
Diabetic retinopathy among patients assisted by a multidisciplinary program at the \\ University Hospital of Ribeirão Preto, São Paulo - USP
}

Eduardo Vieira de Souza ${ }^{1}$

Nivaldo V. de Souza ${ }^{2}$

Maria de Lourdes Veronese Rodrigues ${ }^{3}$

\begin{tabular}{|l|}
\hline RESUMO \\
\hline Objetivo: Analisar o perfil oftalmológico dos pacientes com diabetes \\
melito atendidos pelo programa de atendimento multidisciplinar do Hospi- \\
tal das Clínicas da Faculdade de Medicina de Ribeirão Preto - USP. \\
Métodos: Foi realizada a análise dos prontuários oftalmológicos de 2.360 \\
pacientes atendidos entre 1983 e 2002, por um único médico oftalmologista \\
de um hospital público. Todos os pacientes participavam de um programa \\
de atendimento multidisciplinar, tendo sido encaminhados do Ambulató- \\
rio de Endocrinologia com diagnóstico confirmado de diabetes. Os grupos \\
foram divididos de acordo com o tempo de seguimento em: grupo A - 0 a \\
4, grupo B - 5 a 9, grupo C - 10 a 14 e grupo D - 15 a 19 anos. Resultados: \\
a freqüência de retinopatia diabética por grupo foi de: grupo A - 16\%, B - \\
29\%, C - 36\% e D - 44\%. Houve diferença estatisticamente significante \\
entre o grupo A e os demais (p<0,05) e não houve diferença entre os grupos \\
B, C e D (p>0,05). Conclusão: Mesmo dentro de uma estrutura organizada \\
e com atendimento multidisciplinar visando melhor atenção aos pacientes \\
com diabetes, a retinopatia diabética permanece como complicação freqüen- \\
te e de difícil controle entre os diabéticos. \\
\hline
\end{tabular}

Descritores: Retinopatia diabética/epidemiologia; Programas médicos regionais; Prestação de cuidados de saúde; Hospitais públicos

Otorrinolaringologia e Cirurgia de Cabeca e Pescoço do Hospital das Clínicas da Faculdade de Medicina de Ribeirão Preto da Universidade de São Paulo - USP.

${ }^{1}$ Pós-graduando da Faculdade de Medicina de Ribeirão Preto da Universidade de São Paulo - USP.

2 Professor Doutor do Departamento de Oftalmologia, Otorrinolaringologia e Cirurgia de Cabeça e Pescoço do Hospital das Clínicas da Faculdade de Medicina de Ribeirão Preto da Universidade de São Paulo - USP.

3 Professora Associada do Departamento de Oftalmologia, Otorrinolaringologia e Cirurgia de Cabeça e Pescoço do Hospital das Clínicas da Faculdade de Medicina de Ribeirão Preto da Universidade de São Paulo - USP.

Endereço para correspondência: Eduardo Vieira de Souza, Rua Eliseu Guilherme, 678 - Ribeirão Preto (SP) CEP 14025-020.

E-mail: esmvsouza@yahoo.com.br

Recebido para publicação em 13.11.2002

Versão revisada recebida em 11.04.2003 Aprovação em 04.02.2004

Nota Editorial: Pela análise deste trabalho e por sua anuência na divulgação desta nota, agradecemos ao Dr. Pedro Paulo de Oliveira Bonomo.

\begin{abstract}
$\frac{\text { INTRODUÇÃ̃O }}{\text { O diabetes melito (DM) é uma doença com características sistêmicas que }}$ ocorre em cerca de 6 a $7 \%$ da população brasileira ${ }^{(1)}$ e se caracteriza por causar lesões microangiopáticas, acometendo, principalmente, órgãos como o olho, rim e sistema nervoso periférico. O DM é classificado, principalmente, em diabetes melito insulino-dependente (DMID ou tipo 1), que representa aproximadamente $20 \%$ dos casos e diabetes melito não insulinodependente (DMNID ou tipo 2) com aproximadamente $80 \%$ dos casos. Outros tipos de diabetes como os secundários e gestacionais apresentam uma freqüência muito baixa ${ }^{(2)}$.

O DM está entre as principais causas de cegueira irreversível no Brasil ${ }^{(3)}$ e no mundo ${ }^{(4)}$. As complicações oculares são freqüentes e graves no paciente diabético, e a retinopatia diabética (RD) é a mais comum ${ }^{(4)}$, presente em 29 a $40 \%$ dos doentes diabéticos ${ }^{(5)}$. Vários estudos têm mostrado que quanto maior o tempo de aparecimento da doença, maior a freqüência de retinopatia $^{(6)}$. Pacientes com diabetes tipo II há mais de 15 anos desenvolvem RD
\end{abstract}


em $50 \%$ dos casos, enquanto que aqueles com mais de 25 anos, $80 \%$ apresentam lesões retinianas secundárias ao diabetes $^{(7)}$. Além do tempo de evolução, o controle dos níveis glicêmicos sangüíneos é outro fator importante para boa evolução da doença ${ }^{(8-9)}$.

Estudos têm mostrado que o tratamento da RD é efetivo na prevenção da perda visual ${ }^{(10-11)}$, e que a detecção precoce e acompanhamento são importantes para o bom prognóstico. Estudos epidemiológicos têm fornecido informações valiosas sobre a prevalência e distribuição da RD em diversas populações ${ }^{(6,12)}$, o que é muito útil para identificação de subgrupos de risco e para o planejamento da saúde pública. Entretanto, a maioria destes estudos só mostra que o problema existe, fornecendo poucos dados sobre como preveni-lo ou tratá-lo.

Com o intuito de oferecer um melhor atendimento a estes pacientes diabéticos, em 1983 foi criado no Hospital das Clínicas de Ribeirão Preto um Programa de Atendimento Multidisciplinar (endocrinologia, oftalmologia, neurologia e nefrologia). O objetivo deste programa foi oferecer diagnóstico e tratamento precoces das complicações do diabetes, principalmente oftalmológicas, proporcionando melhor qualidade de vida a estes pacientes.

Este primeiro trabalho tem por objetivo analisar o perfil oftalmológico dos pacientes com diabetes atendidos pelo programa de atendimento Multidisciplinar do Hospital das Clínicas da Faculdade de Medicina de Ribeirão Preto - USP.

\section{MÉTODOS}

Foram estudados de forma retrospectiva os prontuários de 2.360 pacientes do ambulatório de RD do Hospital das Clínicas da Faculdade de Medicina de Ribeirão Preto-USP, atendidos no período de 1983 a 2002 e provenientes da região de Ribeirão Preto-SP, zona urbana ou rural. A grande maioria destes indivíduos estudados tinha baixo nível socioeconômico, apresentando uma renda familiar igual ou menor a três salários mínimos regionais. Os pacientes foram avaliados, inicialmente, por endocrinologistas do ambulatório de clínica médica que encaminharam todos os pacientes com diagnóstico confirmado de diabetes para exame oftalmológico. Este diagnóstico se fez de acordo com o National Diabetes Data Group ${ }^{(13)}$. Os grupos foram subdivididos de acordo com o tempo de acompanhamento em: grupo A (0 a 4 anos); grupo B (5 a 9 anos); grupo C (10 a 14 anos) e grupo D (15 a 19 anos).

O exame oftalmológico contava com medida da acuidade visual com melhor correção, biomicroscopia e tonometria de aplanação, seguido pela instilação de tropicamida $1 \%$ (Mydriacy $^{\circledR}$ ) e de cloridrato de fenilefrina $10 \%$ (Fenilefrina ${ }^{\circledR}$ ). Após aproximadamente vinte minutos realizava-se o exame de fundo de olho através da oftalmoscopia binocular indireta e biomicroscopia de fundo e, quando necessário, angiografia fluoresceínica. Todos os pacientes foram examinados por um único médico retinólogo, um dos autores, e os retornos eram agendados anualmente para pacientes com fundo de olho normal ou períodos menores conforme o caso.
Foram considerados normais pacientes sem alterações oftalmoscópicas visíveis ou menos de seis hemorragias puntiformes. Foram considerados portadores de retinopatia diabética não proliferativa (RDNP) pacientes com, no mínimo, seis ou mais microaneurismas e/ou microhemorragias, e de retinopatia diabética proliferativa (RDP) pacientes com áreas de neovascularização retiniana ou papilar.

Utilizou-se para análise estatística o teste qui-quadrado com correção de Yates do programa Epi-info 6.0 para comparação das porcentagens, adotando-se como significativo o valor de 5\%. Os resultados são expressos como média \pm desviopadrão ou mediana e amplitude de valores observados.

\section{RESULTADOS}

Dos 2.360 pacientes, $1.533(65,0 \%)$ eram do sexo feminino e $827(35,0 \%)$ do masculino. Com relação à classificação, 1.978 $(83,8 \%)$ foram classificados em DM tipo II e $382(16,2 \%)$ em DM tipo I. A idade média foi de 49,3 anos (desvio padrão: $\pm 18,5$ anos, variação de 4 meses a 97 anos).

A prevalência de RD foi observada em $20 \%$ dos pacientes, sendo que $12 \%$ desenvolveram RDNP e $8 \%$ RDP. O número de pacientes por grupo, bem como a prevalência de RD e análise estatística estão dispostos na Tabela 1 . O gráfico 1 mostra a

\begin{tabular}{|c|c|c|c|c|}
\hline \multicolumn{5}{|c|}{$\begin{array}{l}\text { Tabela } 1 \text { - Análise estatística e porcentagem de retinopatia diabética } \\
\text { dividida por grupo, de acordo com o tempo de seguimento em } 2.360 \\
\text { pacientes atendidos no Hospital das Clínicas da Faculdade de } \\
\text { Medicina de Ribeirão Preto - USP. Grupo A ( } 0 \text { a } 4 \text { anos), grupo B (5 } \\
\text { a } 9 \text { anos), grupo C (10 a } 14 \text { anos) e grupo D (15 a } 19 \text { anos). Cálculo } \\
\text { estatístico pelo teste do qui-quadrado }\end{array}$} \\
\hline & Grupo A & Grupo B & Grupo C & Grupo D \\
\hline Pacientes & 1.740 & 402 & 166 & 52 \\
\hline RD & $16 \%$ & $29 \%$ & $36 \%$ & $44 \%$ \\
\hline Estatística & $p<0,05$ & $p>0,05$ & $p>0,05$ & $p>0,05$ \\
\hline \multicolumn{5}{|c|}{ RD - Retinopatia diabética } \\
\hline
\end{tabular}

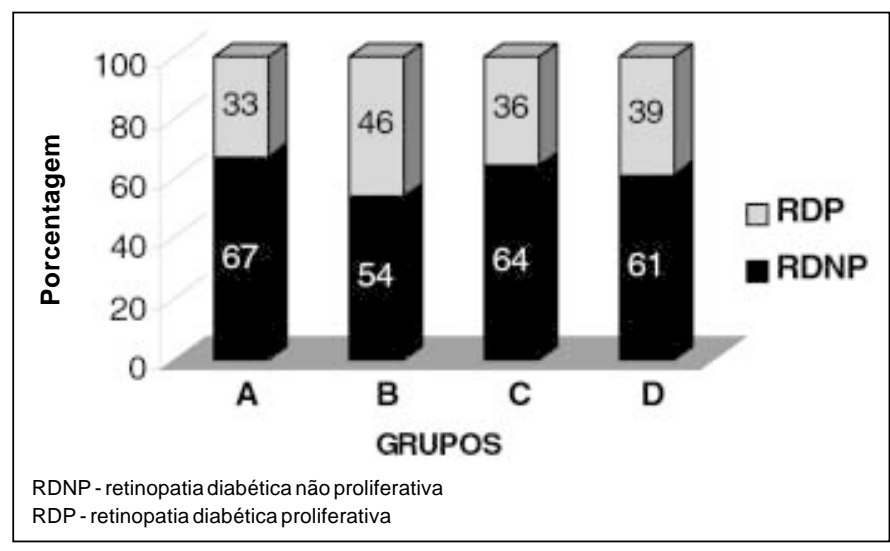

Gráfico 1 - Tipo de retinopatia diabética em porcentagem, dividido por grupo, de acordo com o tempo de seguimento em 2.360 pacientes atendidos no Hospital das Clínicas da Faculdade de Medicina de Ribeirão Preto - USP. Grupo A ( 0 a 4 anos), grupo B (5 a 9 anos), grupo $C$ (10 a 14 anos) e grupo $D$ (15 a 19 anos) 
porcentagem do tipo de RD por grupo. Com relação ao grupo A, 974 pacientes foram atendidos uma única vez, sendo que destes 747 perderam o seguimento.

\section{DISCUSSÃO}

Uma das grandes dificuldades quando se faz estudos epidemiológicos com pacientes diabéticos reside em determinar o tempo de instalação da doença ${ }^{(7)}$. Diversos trabalhos ${ }^{(4-6)}$ depararam com a mesma dificuldade, tentando de forma paliativa contornar o problema. A decisão foi, então, dividir os grupos de acordo com o tempo de seguimento, uma vez que não se dispunha de dados confiáveis a respeito do tempo de instalação da doença. Desta forma, se conseguiu traçar um bom perfil destes pacientes, o que permitiu uma boa análise do programa de atendimento multidisciplinar desenvolvido pelo hospital. Dados como medicação para controle da hiperglicemia e presença de hipertensão arterial sistêmica não foram apresentados por não constarem dos prontuários oftalmológicos destes pacientes. Estes e outros dados seriam importantes para melhor caracterização deste grupo.

A prevalência geral de retinopatia diabética neste estudo se mostrou semelhante a outro estudo ${ }^{(14)}$, que encontrou $24 \%$ de retinopatia, sendo que destes $65 \%$ nunca haviam sido submetidos a exame de oftalmoscopia. Da mesma forma, encontrou-se um grande número de pacientes com retinopatia atendidos na primeira consulta. Por outro lado, vários foram os estudos que registraram uma prevalência de retinopatia superior a nossa ${ }^{(15-17)}$, com variações de $34 \%$ a $65 \%$. Apesar de bem estruturados, os estudos nacionais são realizados, em geral, com amostras pequenas e viciadas. $\mathrm{O}$ presente estudo utilizou uma amostra significativa, com um tempo de seguimento prolongado. Entretanto a amostra, da mesma forma que diversos outros estudos, se mostrou viciada uma vez que utilizou somente pacientes da faixa econômica mais pobre da população.

Com relação à prevalência de RD nos diferentes grupos, foi observada uma diferença estatisticamente significante entre o grupo A e os outros grupos ( $\mathrm{p}<0,05)$. Entre os grupos B, C e D não encontramos diferença estatística $(p>0,05)$. Acreditamos que estes dados tenham sido gerados por dois motivos: grande número de pacientes no grupo A com um único atendimento, sendo que muitos deles perderam seguimento; e os pacientes com RD mostraram maior adesão ao tratamento, atendendo com maior empenho aos retornos.

Por outro lado, observou-se um aumento percentual destes valores (grupo A 16\%, grupo B 29\%, grupo C $36 \%$ e grupo D 44\%). Entretanto, observados os valores absolutos, nota-se uma redução nestes números, com diminuição importante do seguimento (grupo A - 272, grupo B - 116, grupo C - 59 e grupo D - 23 pacientes). Acredita-se que fatores como óbito, mudança de cidade e dificuldade de locomoção tenham colaborado para isso.
O grupo A foi o grupo com maior número de pacientes (1.740 pacientes). Destes, mais da metade (974 pacientes), foram atendidos uma única vez. Apesar disso, a prevalência da RD no grupo A se mostrou alta (16\%) se comparada a alguns estudos ${ }^{(18-19)}$, e baixa se comparada a outros ${ }^{(20,21,22)}$.

Outro dado importante diz respeito à presença de RDP, que não se instala em pacientes com diabetes tipo I antes de 3 a 5 anos de doença. Nos diabéticos tipo II o tempo para instalação é ainda maior, em torno de 5 a $7 \operatorname{anos}^{(18)}$. Encontrou-se RDP em $40 \%$ dos pacientes com RD do grupo A. Estes dados permitem inferir que: o diabetes está sendo diagnosticada tardiamente; ou os pacientes estão sendo encaminhados tardiamente ao oftalmologista.

\section{CONCLUS ÃO}

Mesmo dentro de uma estrutura organizada e com atendimento multidisciplinar visando uma melhor atenção aos pacientes com diabetes, a retinopatia diabética permanece como uma complicação freqüente e de difícil controle entre os diabéticos.

\section{ABSTRACT}

Purpose: To analyze the ophthalmological profile of diabetic patients assisted by the multidisciplinary program at the Clinical Hospital of Ribeirão Preto-USP. Methods: The analysis of the records of fundus ophthalmoscopy, performed by the same ophthalmologist, was carried out in 2.360 diabetic patients from 1983 to 2002, who were divided according to the followup period: group A - 0 to 4, group B - 5 to 9, group C - 10 to 14 and group D - 15 to 19 years. Results: The frequency of retinopathy was: A $-16 \%, \mathrm{~B}-29 \%, \mathrm{C}-36 \%$ and D $-44 \%$. There were significant differences between $\mathrm{A}$ and the other groups $(\mathrm{p}<0,05)$ and no differences between B, C and D ( $>>0,05)$. Conclusion: Even with an organized and multidisciplinary care, diabetic retinopathy continues to be a frequent and hard control disease among diabetic patients.

Keywords: Diabetic retinopathy/epidemiology; Regional medical programs, Delivery of health care; Hospitals, public

\section{REFERÊNCIAS}

1. Moreira Jr. CA, Ávila MP. In: Conselho Brasileiro de Oftalmologia. Manual de retina e vítreo. Rio de Janeiro: Cultura Médica; 2000. p.61-76.

2. Foster D. Diabetes melito. In: Harrison, Medicina interna. 13ำ ed. Rio de Janeiro: Guanabara-Koogan; 1995. p.2079-101.

3. Ministério da Saúde. Brasil.Diabetes melito. Guia básico para diagnóstico e tratamento. Brasília; Sociedade Brasileira de Diabetes; 1996. p.5, 7, 57. [Programa Harvard/ Joslin/ SBD].

4. National Society to Prevent Blindness. Operation Research Dept. Vision problems in the U.S.: a statistical analysis. New York: The Society; 1980.

5. Klein BE, Klein R, Wang Q, Moss SE. Older-onset diabetes and lens opacities. The Beaver Dam Eye Study. Ophthalmic Epidemiol 1995;2:49-55. 
6. Aldington SJ, Kohner EM, Meuer S, Klein R, Sjolie AK. Methodology for retinal photography and assessment of diabetic retinopathy: The EURODIAB IDDM Complications Study. Diabetologia 1995;38:437-44.

7. Souza NV. Retina. In: Rodrigues MLV coordenador. Oftalmologia clínica. Rio de Janeiro: Cultura Médica; 1992. p.230.

8. The effect of intensive treatment of diabetes on the development and progression of long-term complications in insulin dependent diabetes mellitus. The Diabetes Control and Complications Trial Research Group. N Engl J Med 1993;329:977-86.

9. Progression of retinopathy with intensive versus conventional treatment in the Diabetes Control and Complications Trial. Diabetes Control and Complications Trial Research Group. Ophthalmology 1995;102:647-61.

10. Indications for photocoagulation treatment of diabetic retinopathy: Diabetic Retinopathy Study Report no. 14. The Diabetic Retinopathy Study Research Group. Int Ophthalmol Clin 1987;27:239-53.

11. Photocoagulation for diabetic macular edema. Early Treatment Diabetic Retinopathy Study: Report number 1. Early Treatment Diabetic Retinopathy Study research group. Arch Ophthalmol 1985;103:1796-806.

12. Kini MM, Leibowitz HM, Colton T, Nickerson RJ, Ganley J, Dawber TR. Prevalence of senile cataract, diabetic retinopathy, senile macular degeneration and open angle glaucoma in the Framingham eye study. Am J Ophthalmol 1978;85:28-34.

13. Classification and diagnosis of diabetes mellitus and other categories of glucose intolerance. National Diabetes Data Group. Diabetes 1979;28:1039-57.

14. Ramos SR, Sabbag FP, Busato D, Miranda AB, Moreira Júnior CA. Reti- nopatia diabética: estudo de uma associação de diabéticos. Arq Bras Oftalmol 1999; 62:735-7.

15. Garcia CAA, Oliveira TL, Andrade BBA, Nunes IM, Silva JM da. Incidência e fatores de risco da retinopatia diabética em pacientes do Hospital Universitário Onofre Lopes [abstract]. Arq Bras Oftalmol 1999;62:439.

16. Moura CAA, Fernandes MLPM, Jorge PT. Estudo da prevalência da retinopatia diabética no ambulatório da Universidade Federal de Uberlândia [abstract]. Arq Bras Oftalmol 1996;59:349.

17. Steck AD, Esteves RF, Gonçalves JCM. Prevalência de retinopatia diabética em uma população de diabéticos do HC de Franco da Rocha - SP. Arq Bras Oftalmol 1993;56:125-8.

18. Klein R, Klein BE, Moss SE, Linton KL. The Beaver Dam Eye Study. Retinopathy in adults with newly discovered and previously diagnosed diabetes mellitus. Ophthalmology 1992;99:58-62.

19. Klein R, Barrett-Connor EL, Blunt BA, Wingard DL. Visual impairment and retinopathy in people with normal glucose tolerance, impaired glucose tolerance, and newly diagnosed NIDDM. Diabetes Care 1991;14:914-8.

20. Klein R, Klein BE, Moss SE, Davis MD, DeMets DL. The Wisconsin epidemiologic study of diabetic retinopathy. III. Prevalence and risk of diabetic retinopathy when age at diagnosis is 30 or more years. Arch Ophthalmol 1984;102:527-32.

21. Mc Kay R, McCarty CA, Taylor HR. Diabetic retinopathy in Victoria, Australia: the Visual Impairment Project. Br J Ophthalmol 2000;84:865-70.

22. Mitchell P, Smith W, Wang JJ, Attebo K. Prevalence of diabetic retinopathy in an older community: The Blue Mountains Eye Study. Ophthalmology 1998;105:406-11.

\title{
XXIX Simpósio da Associação Paranaense de Oftalmologia
}

\author{
24 a 26 de Junho de 2004 \\ Bourbon Curitiba Hotel \\ CURITIBA - PR
}

INFO RMAÇÕES: JDE Comunicação e Eventos

Tels.: (11) 5082-3030 / 5084-5284

Fax: (11) 5574-8261

E-mail: jdecomev@uol.com.br 